\title{
CD19-dependent B lymphocyte signaling thresholds influence skin fibrosis and autoimmunity in the tight-skin mouse
}

\author{
Eriko Saito, ${ }^{1}$ Manabu Fujimoto, ${ }^{2}$ Minoru Hasegawa, ${ }^{1}$ Kazuhiro Komura, ${ }^{1}$ \\ Yasuhito Hamaguchi, ${ }^{1}$ Yuko Kaburagi, ${ }^{1}$ Tetsuya Nagaoka, ${ }^{1}$ Kazuhiko Takehara, ${ }^{1}$ \\ Thomas F. Tedder, ${ }^{3}$ and Shinichi Sato ${ }^{1}$
}

${ }^{1}$ Department of Dermatology, Kanazawa University Graduate School of Medical Science, Kanazawa, Japan
${ }^{2}$ Department of Regenerative Medicine, Research Institute, International Medical Center of Japan, Tokyo, Japan
${ }^{3}$ Department of Immunology, Duke University Medical Center, Durham, North Carolina, USA

Address correspondence to: Shinichi Sato, Department of Dermatology, Kanazawa University Graduate School of Medical Science, 13-1 Takaramachi, Kanazawa, Ishikawa 920-8641, Japan.

Phone: 81-76-265-2341; Fax: 81-76-234-4270; E-mail: s-sato@med.kanazawa-u.ac.jp.

Received for publication January 18, 2002, and accepted in revised form April 16, 2002.

\begin{abstract}
The tight-skin (TSK/+) mouse, a genetic model for human systemic sclerosis (SSc), develops cutaneous fibrosis and autoantibodies against SSc-specific target autoantigens. Although molecular mechanisms explaining the development of fibrosis and autoimmunity in SSc patients or TSK/+ mice remain unknown, we recently demonstrated that SSc patients overexpress CD19, an important regulatory molecule expressed by B lymphocytes. B cells from CD19-deficient mice are hyporesponsive to transmembrane signals, while $\mathrm{B}$ cells overexpressing CD19 are hyperresponsive and generate autoantibodies. In this study, TSK/+ B cells also exhibited a hyperresponsive phenotype with decreased surface IgM expression, enhanced serum Ig production, and spontaneous autoantibody production. Moreover, CD19 tyrosine phosphorylation was constitutively augmented in TSK/+ $\mathrm{B}$ cells. CD19-mediated $\left[\mathrm{Ca}^{2+}\right]_{\mathrm{i}}$ responses, Vav phosphorylation, and Lyn kinase activity were similarly enhanced. Studies of TSK/+ mice deficient in CD19 expression demonstrated that CD19 deficiency significantly decreased skin fibrosis in TSK/+ mice. Additionally, CD19 loss in TSK/+ mice upregulated surface IgM expression and completely abrogated hyper- $\gamma$-globulinemia and autoantibody production. CD19 deficiency also inhibited IL- 6 production by TSK/+ B cells. Thus, chronic B cell activation resulting from augmented CD19 signaling in TSK/+ mice leads to skin sclerosis possibly through IL-6 overproduction as well as autoimmunity.
\end{abstract}

J. Clin. Invest. 109:1453-1462 (2002). doi:10.1172/JCI200215078.

\section{Introduction}

Systemic autoimmune diseases exhibit complex symptoms that involve multiple organs and cell types. Systemic sclerosis (SSc) is a connective tissue disease characterized by excessive ECM deposition in the skin and other visceral organs. Although the molecular basis for SSc is unknown, the presence of highly disease-specific autoantibodies, including anti-DNA topoisomerase I (topo I) Ab's, suggests an autoimmune component to disease pathogenesis. However, a relationship between autoimmunity and fibrosis remains unclear. The tightskin (TSK) mouse is a genetic model for human SSc. While the phenotypic characteristics of TSK mice are not identical to those of human SSc patients, TSK mice produce autoantibodies against SSc-specific target autoantigens including topo I, fibrillin 1 (Fbn-1), RNA polymerase I, collagen type I, and Fc $\gamma$ receptors (1-3). The TSK mouse was originally identified (4) as a spontaneous mutation exhibiting increased synthesis and accumulation of collagen and other ECM proteins in the skin. Although homozygous mice die in utero, het- erozygous (TSK/+) mice survive but develop cutaneous fibrosis. TSK/+ mice also have pulmonary emphysema and cardiac hypertrophies (4). A tandem duplication within the Fbn-1 gene is suggested to cause the TSK phenotype $(5,6)$. Fbn- 1 is the major structural protein of a widely distributed class of connective tissue microfibrils that are key components of elastic fibers. Although the duplicated Fbn-1 gene gives rise to an oversized Fbn-1 protein that results in abnormal microfibrils $(7,8)$, a recent study has demonstrated increased proteolysis of the abnormal microfibrils (7). Therefore, the phenotypic consequences of the duplicated Fbn-1 gene may only account for lung emphysema (7). Moreover, transgenic mice expressing a mutated Fbn-1 gene develop cutaneous hyperplasia, but not pulmonary emphysema and myocardial hypertrophy (9). Thus, the role of the Fbn-1 gene in the genesis of tissue hyperplasia and autoimmunity remains unresolved.

Crucial features of most autoimmune diseases include autoantibody production and skewed humoral immune responses. Humoral immune responses are 
normally regulated by signal transduction molecules that amplify or inhibit B cell antigen receptor (BCR) signaling during responses to self and foreign antigens (10). These regulatory molecules include a subset of functionally interrelated cell surface receptors, such as CD19 and CD22, and their intracellular signaling components, including Lyn protein tyrosine kinase and the SHP-1 protein tyrosine phosphatase (10). Altered function or expression of these molecules can influence susceptibility for autoimmunity. For example, Lyn-deficient mice exhibit glomerulonephritis due to the presence of immune complexes containing autoantibodies (11). Motheaten viable $\left(m e^{v} / m e^{v}\right)$ mice with SHP-1 mutations produce elevated levels of spontaneous autoantibodies, hyper- $\gamma$-globulinemia, and tissue deposition of immune complexes (12). Transgenic mice that overexpress CD19 approximately threefold lose tolerance and generate autoantibodies spontaneously (13, 14). Autoantibodies are also found in transgenic mice with subtle increases of about 20\% in CD19 expression (15). Remarkably, similar increases in CD19 expression are found on B cells from patients with SSc, with CD19overexpressing mice and SSc patients producing characteristic autoantibodies with similar specificities (15). Therefore, increased CD19 expression and signaling in $\mathrm{B}$ cells may contribute to autoimmunity in SSc patients.

Since CD19 is a general "rheostat" that defines signaling thresholds critical for humoral immune responses and autoimmunity (10) and CD19 expression is increased in patients with SSc (15), it was assessed whether there were also abnormalities in CD19 expression or function in the TSK mouse model of SSc. Remarkably, TSK/+ B cells displayed a hyperresponsive phenotype, with enhanced CD19-induced $\left[\mathrm{Ca}^{2+}\right]_{\mathrm{i}}$ responses and higher levels of constitutive CD19 tyrosine phosphorylation. To further determine an in vivo role of CD19 signaling in the development of autoimmunity and skin sclerosis in TSK/+ mice, TSK/+ mice deficient in CD19 expression (CD19-/-TSK/+ mice) were generated. In addition, the loss of CD19 expression significantly downregulated the hyperresponsive phenotype in TSK/+ B cells and eliminated autoantibody production. CD19 deficiency also inhibited the development of skin fibrosis in TSK/+ mice. Thus, alterations in CD19-dependent signaling pathways contribute to both skin fibrosis and systemic autoimmunity in TSK/+ mice.

\section{Methods}

Mice. $\mathrm{CD} 19^{-/-}(\mathrm{C} 57 \mathrm{BL} / 6 \times 129)$ mice were generated as described (16) and backcrossed between 7 and 12 generations onto the C57BL/ 6 background before use in this study. TSK/+ mice with a C57BL/6 genetic background were purchased from The Jackson Laboratory (Bar Harbor, Maine, USA). CD19-/-, TSK/+, CD19-/-TSK/+, and wild-type littermates were generated by crossing $C D 19^{+/-T S K /+}$ parents. Lack of cell surface CD19 expression was verified by two-color immunofluorescence staining with flow cytometric analysis. To verify the TSK/+ genotype, PCR amplification of a partially duplicated Fbn-1 gene was carried out using genomic DNA from each mouse as described (17). All mice were housed in a specific pathogen-free barrier facility and screened regularly for pathogens. Mice used in these experiments were 3 months of age. All studies and procedures were approved by the Committee on Animal Experimentation of Kanazawa University Graduate School of Medical Science.

$A b$ 's and flow cytometric analysis. Ab's used in this study included mouse IgA anti-mouse CD19 (MB19-1) mAb (13); biotinylated or FITC-conjugated anti-IgM (Southern Biotechnology Associates Inc., Birmingham, Alabama, USA); biotinylated or FITC-conjugated anti-B220 (BD PharMingen, San Diego, California, USA); phycoerythrin-conjugated (PE-conjugated) antiCD5 (BD PharMingen); and PE-conjugated anti-CD23 Ab's (BD PharMingen). PE-conjugated streptavidin (Southern Biotechnology Associates Inc.) was used to reveal staining with biotin-coupled Ab's. For two-color immunofluorescence analysis, single-cell suspensions of lymphocytes from blood, spleen, bone marrow, and peritoneal lavage were used. Leukocytes $\left(0.5 \times 10^{6}\right.$ to $\left.1 \times 10^{6}\right)$ were stained at $4^{\circ} \mathrm{C}$ using predetermined optimal concentrations of Ab's for 20 minutes as described (18). Cells with the forward and side light scatter properties of lymphocytes were analyzed on a FACScan flow cytometer (BD PharMingen).

$B$ cell purification, immunoprecipitations, and Western blot analysis. Splenic B cells were purified $\left(>95 \% \mathrm{~B} 220^{+}\right)$by removing $\mathrm{T}$ cells with anti-Thy $1.2 \mathrm{Ab}$-coated magnetic beads (Dynal Inc., Lake Success, New York, USA), and subsequently lysed in buffer containing $1 \%$ Nonidet P-40 as described (19). For CD19 immunoprecipitations, the lysates were incubated with Affigel-10 beads (Bio-Rad Laboratories Inc., Hercules, California, USA) bearing anti-CD19 mAb for 4 hours at $4^{\circ} \mathrm{C}$. For Vav immunoprecipitations, the cell lysates were incubated with protein G-Sepharose beads plus anti-Vav Ab (Santa Cruz Biotechnology Inc., Santa Cruz, California, USA). Immunoprecipitated proteins were subjected to SDS-PAGE and transferred onto membranes for immunoblotting. These membranes were incubated with horseradish peroxidase-conjugated anti-phosphotyrosine Ab (PY99; Santa Cruz Biotechnology Inc.). These blots were developed using an enhanced chemiluminescence kit (Pierce Chemical Co., Rockford, Illinois, USA). To verify equivalent amounts of protein in each lane, the blots were stripped and reprobed with Ab's against Vav or CD19 (Santa Cruz Biotechnology Inc.). Band intensities were quantified using Quantity One software (Bio-Rad Laboratories Inc.).

In vitro Lyn kinase assays. Splenic B cell lysates were precleared and incubated with anti-Lyn Ab's (Santa Cruz Biotechnology Inc.) and protein A-Sepharose beads for 3 hours at $4^{\circ} \mathrm{C}(19)$. The beads were incubated with 10 $\mu \mathrm{g}$ of cdc2 (6-20) $\mathrm{NH}_{2}$ peptide (Upstate Biotechnology Inc., Lake Placid, New York, USA) and $10 \mu \mathrm{Ci}$ of [ $\left.\gamma^{-32} \mathrm{P}\right]$ ATP for 2 minutes at $25^{\circ} \mathrm{C}$. The reactions were termi- 
nated by adding $40 \%$ trichloroacetic acid and spotted onto p81 phosphocellulose paper. Radioactivity was quantified by scintillation counting.

Measurement of $\left[\mathrm{Ca}^{2+}\right]_{i}$. Isolated splenocytes were loaded with $1 \mu \mathrm{M}$ indo- 1 AM ester (Molecular Probes Inc., Eugene, Oregon, USA) at $37^{\circ} \mathrm{C}$ for 30 minutes, and then stained with FITC-conjugated anti-B220 Ab. For analysis, the ratio of fluorescence $(525 / 405 \mathrm{~nm})$ for $\mathrm{B} 220^{+}$cells was determined using an Epics Altra cell sorter (Beckman Coulter Inc., Miami, Florida, USA). Base-line fluorescence ratios were collected for 1 minute before addition of anti-CD19 mAb $(40 \mu \mathrm{g} / \mathrm{ml})$. Fluorescence ratios were obtained in real time for 7 minutes following $\mathrm{mAb}$ addition. An increase in the fluorescence ratio indicates an increase in $\left[\mathrm{Ca}^{2+}\right]_{\mathrm{i}}$.

$B$ cell proliferation. Purified spleen B cells were cultured in $0.2 \mathrm{ml}$ of culture medium in 96-well flat-bottom plates with LPS (Sigma-Aldrich, St. Louis, Missouri, USA), $\mathrm{F}\left(\mathrm{ab}^{\prime}\right)_{2}$ anti-mouse IgM Ab's (Cappel, Durham, North Carolina, USA), or anti-CD38 mAb (Ab90; BD PharMingen) for 72 hours. Proliferation was assessed by the incorporation of $\left[{ }^{3} \mathrm{H}\right]$-labeled thymidine $(1$ $\mu \mathrm{Ci} /$ well) added during the last 16 hours of culture, followed by scintillation counting. All treatments were carried out in triplicate cultures.

Antinuclear Ab analysis. Antinuclear Ab's (ANAs) were assessed by indirect immunofluorescence staining using sera diluted 1:50 and HEp-2 substrate cells (Medical \& Biological Laboratories Co., Nagoya, Japan) as described (15). ANAs were detected using FITC-conjugated $\mathrm{F}\left(\mathrm{ab}^{\prime}\right)_{2}$ fragments specific for mouse IgG+IgM+IgA (Southern Biotechnology Associates Inc.).

ELISAs for autoantibodies. Serum autoantibody levels were determined by ELISA as described (15). Briefly, 96well microtiter plates were coated with single-stranded DNA (ssDNA; Sigma-Aldrich), double-stranded DNA (dsDNA; Medical \& Biological Laboratories Co.), histone (Sigma-Aldrich), the recombinant human Fbn-1 peptide, or rabbit IgG (Sigma-Aldrich). Plates were incubated with serum samples diluted 1:100. The bound $\mathrm{Ab}$ 's were detected with alkaline phosphatase-conjugated anti-mouse IgG or IgM Ab's (Southern Biotechnology Associates Inc.). Anti-topo I Ab's were measured with specific ELISA kits (Medical $\&$ Biological Laboratories Co.). The recombinant peptide of the proline-rich $C$ region (amino acids 395-446) of Fbn-1 was generated as described elsewhere $(2,3)$. Relative levels of autoantibodies were determined for each group of mice using pooled serum samples. Sera were diluted at log intervals $\left(1: 10-1: 10^{5}\right)$ and assessed for relative autoantibody levels as above except that the results were plotted as OD versus dilution (log scale). The dilutions of sera giving half-maximal OD values were determined by linear regression analysis, thus generating arbitrary unit per milliliter values for comparison between sets of sera.

Mouse Ig isotype-specific ELISAs. To determine Ab concentrations in sera, ELISAs were carried out as described (16), using affinity-purified mouse IgM,
IgG1, IgG2a, IgG2b, IgG3, and IgA (Southern Biotechnology Associates Inc.) to generate standard curves. The relative Ig concentration of individual samples was calculated by comparing the mean OD obtained for duplicate wells to a semi-log standard curve of titrated standard $\mathrm{Ab}$ using linear regression analysis.

Histopathological assessment of skin fibrosis. Morphologic characteristics of skin sections from $\mathrm{CD} 19^{-/-} \mathrm{TSK} /+$ mice were compared with those of $\mathrm{CD}_{19} 9^{--}, \mathrm{TSK} /+$, and wild-type littermates under a light microscope. All skin sections were taken from the para-midline, lower back region (the same anatomic site, to minimize regional variations in thickness) as full-thickness sections extending down to the body wall musculature. Tissues were fixed in $10 \%$ formaldehyde solution for 24 hours and embedded in paraffin. Sections were stained with hematoxylin and eosin. Hypodermal thickness, which was defined as the thickness of a subcutaneous loose connective tissue layer (i.e., the hypodermis or superficial fascia) beneath the panniculus carnosus, was measured for multiple transverse perpendicular sections using an ocular micrometer. Dermal thickness, defined as the thickness of skin from the top of the granular layer to the junction between the dermis and subcutaneous fat, was also examined. Ten random measurements were taken per section. All of the sections were examined independently by two investigators in a blinded fashion. The skin from male mice was generally thicker than that from female mice in spite of the presence or absence of TSK mutations (data not shown). Since similar results were obtained when male or female mice were analyzed separately, only data from female mice were presented for skin thickness and hydroxyproline content in this study.

Determination of hydroxyproline content in the skin tissue. Hydroxyproline is a modified amino acid uniquely found as a high percentage of collagen. Six-millimeter punch biopsies from shaved dorsal skin samples were treated with chloroform/methanol $(2: 1 \mathrm{vol} / \mathrm{vol})$ to remove the fat and were dried by centrifugation under vacuum. Dried samples were weighed, and acid hydrolyzed for 24 hours at $110^{\circ} \mathrm{C}$. The hydroxy lysates were dried, redissolved in $200 \mu \mathrm{l}$ of water, and filtered through millipore filters. Sample aliquots of $20 \mu \mathrm{l}$ were diluted tenfold and used for amino acid composition analysis in an Amino Acid Analyzer (Hewlett-Packard, Palo Alto, California, USA).

Production of IL- 6 and IgG1 by splenic B cells. Purified splenic $B$ cells $\left(2 \times 10^{5}\right)$ were cultured in $0.2 \mathrm{ml}$ of culture medium in 96-well flat-bottom plates with $\mathrm{F}\left(\mathrm{ab}^{\prime}\right)_{2}$ antimouse IgM Ab $(1 \mu \mathrm{g} / \mathrm{ml})$ plus anti-CD40 mAb (1C10, 10 $\mu \mathrm{g} / \mathrm{ml}$; R\&D Systems Inc., Minneapolis, Minnesota, USA) for 48 hours. IL- 6 accumulation in the culture medium was measured by ELISA (R\&D Systems Inc.). Purified splenic B cells $\left(1 \times 10^{5}\right)$ were cultured with LPS $(20 \mu \mathrm{g} / \mathrm{ml})$ plus IL-4 $(100 \mathrm{ng} / \mathrm{ml})$ for 5 days. IgG1 secreted into the culture medium was determined by ELISA.

Statistical analysis. All data are expressed as mean values \pm SEM. The Mann-Whitney $U$ test was used for 
a CD19 immunoprecipitation

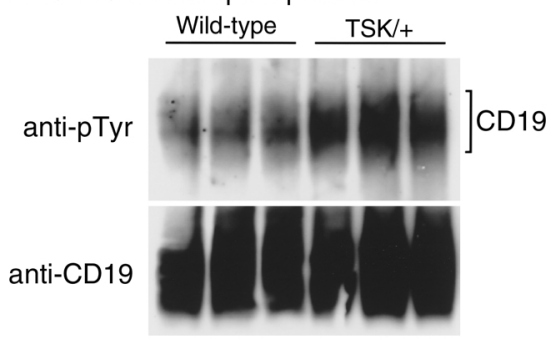

b

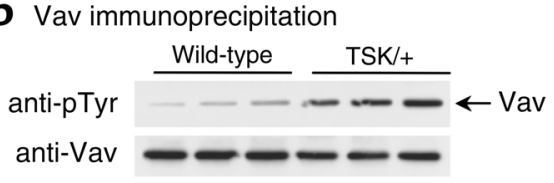

C In vitro Lyn kinase activity

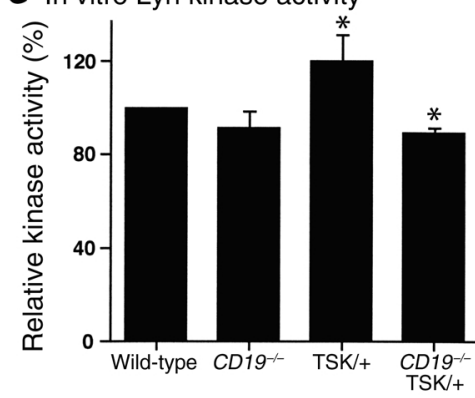

Figure 1

CD19 signal transduction in B cells from TSK/+ and wild-type littermates. (a and b) Constitutive tyrosine phosphorylation of CD19 (a) and $\operatorname{Vav}(\mathbf{b})$ in B cells. Splenic B cells were obtained from three mice of each group. Proteins were immunoprecipitated from lysates of unstimulated B cells with either anti-CD19 or anti-Vav Ab's. Immunoprecipitated proteins were subjected to SDS-PAGE and transferred onto membranes for subsequent anti-phosphotyrosine (anti-pTyr) immunoblotting. All blots were subsequently stripped of anti-pTyr Ab's and reprobed with the precipitating Ab's to verify equivalent amounts of proteins in each lane. Each lane represents results from individual mice but represents results obtained with at least six sets of mice. (c) Constitutive Lyn kinase activity in B cells. Splenic B cells were solubilized and immunoprecipitated with anti-Lyn Ab. Immunoprecipitates were then incubated with cdc2(6-20) $\mathrm{NH}_{2}$ peptide and [ $\left.\gamma-{ }^{32} \mathrm{P}\right]$ ATP. The radioactivity incorporated into cdc2 peptide was quantified by scintillation counting. Relative mean ( \pm SEM) kinase activities are obtained from three experiments. Kinase activity was shown as percentage of wild-type B cells that were defined as $100 \% .{ }^{*} P<0.05$.

determining the level of significance of differences between sample means, and Bonferroni's test was used for multiple comparisons.

\footnotetext{
Results

CD19 expression and signaling in TSK/+ B cells. CD19 expression and signaling were assessed in $B$ cells from $\mathrm{TSK} /+$ mice. Cell surface CD19 expression levels on bone marrow, blood, spleen, and peritoneal TSK/+ B cells were indistinguishable from those found on $\mathrm{B}$ cells from wild-type littermates (data not shown). Similarly, the wild-type amounts of CD19 protein were immunoprecipitated from TSK/+ B cells (Figure 1a). However, constitutive CD19 tyrosine phosphorylation was significantly augmented in splenic TSK/+ B cells compared with wild-type B cells $(45 \%$ increased, $P<0.01$, Figure 1a). Consistent with this, constitutive tyrosine phosphorylation of Vav, a major effector mol-
}

ecule downstream of CD19 signaling $(19,20)$, was also enhanced 3.4-fold in TSK/+ B cells relative to wild-type B cells $(P<0.01$, Figure $1 b)$. Since CD19 regulates Lyn kinase activity $(19,20)$, constitutive in vitro Lyn kinase activity was assessed. Lyn kinase activity was enhanced by $22 \%$ in TSK/+ B cells compared with wild-type B cells $(P<0.05$, Figure $1 \mathrm{c})$. By contrast, CD19 deficiency in TSK/+ B cells inhibited Lyn kinase activity, which was $11 \%$ lower than that found in wild-type B cells $(P<0.05)$. To further assess CD19 signaling in TSK/+ B cells, $\left[\mathrm{Ca}^{2+}\right]_{\mathrm{i}}$ responses generated by CD19 ligation were examined. In wild-type B cells, CD19 engagement using optimal concentrations of a dimeric IgA Ab (40 $\mu \mathrm{g} / \mathrm{ml}$ ) induces a slow rise in $\left[\mathrm{Ca}^{2+}\right]_{\mathrm{i}}$ (Figure 2a). CD19induced $\left[\mathrm{Ca}^{2+}\right]_{\mathrm{i}}$ responses in TSK/+ B cells were significantly augmented when compared with those in wildtype B cells. Proliferation of TSK/+ B cells in response to anti-IgM Ab was significantly increased compared
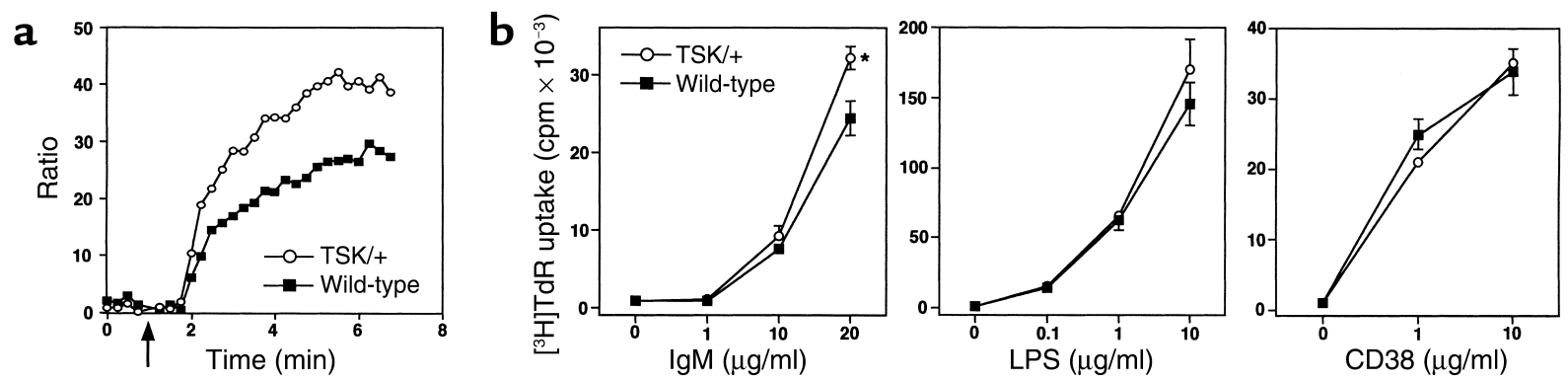

Figure 2

Activation and proliferation of B cells from TSK/+ and wild-type littermates. (a) $\left[\mathrm{Ca}^{2+}\right]_{i}$ responses in B cells following CD19 ligation. Splenocytes were loaded with indo- $1 \mathrm{AM}$ ester and examined for relative $\left[\mathrm{Ca}^{2+}\right]_{\mathrm{i}}$ levels by flow cytometry after gating on the $\mathrm{B} 220^{+}$population of cells. Anti-CD19 mAb was added at the indicated time (arrow). An increase in the fluorescence ratio indicates an increase in [Ca $\left.{ }^{2+}\right]_{i}$. These results represent those obtained in four independent experiments. (b) B cell proliferation in response to anti-IgM Ab's, LPS, and anti-CD38 mAb. Spleen $B$ cells were cultured for 72 hours with the indicated amounts of anti-IgM Ab's, LPS, or anti-CD38 mAb. Values represent the mean cpm ( \pm SEM) of labeled thymidine obtained from triplicate cultures. These results represent those obtained in four independent experiments. ${ }^{*} P<0.05$. 
with wild-type B cells (22\% increase, $P<0.05)$, but $\mathrm{TSK} /+\mathrm{B}$ cells exhibited normal proliferation to LPS or anti-CD38 $\mathrm{mAb}$ (Figure $2 \mathrm{~b}$ ). Thus, the CD19 signaling pathway appeared to be constitutively activated in TSK/+ B cells, which resulted in increased early B cell responses mediated by CD19 and enhanced BCRmediated proliferation.

CD19 deficiency normalizes TSK $/+B$ cell hyperresponsiveness. Constitutively activated $\mathrm{B}$ cells, including $\mathrm{B}$ cells overexpressing CD19, express reduced levels of cell surface IgM as a consequence of augmented signaling (18, $21,22)$. By contrast, CD19-deficient B cells, which are hyporesponsive, express elevated levels of cell surface $\operatorname{IgM}(13,16,22)$. Consistent with a hyperresponsive phenotype of TSK/+ B cells and upregulated CD19 signaling, blood B cells had a 55\% decrease in surface IgM expression relative to $\mathrm{B}$ cells from wild-type littermates $(P<0.0001$, Figure 3$)$. Circulating B cells from $C D 19^{-/-}$ mice demonstrated $137 \%$ higher surface IgM levels than did wild-type B cells $(P<0.01)$. CD19 deficiency in TSK/+ mice resulted in $125 \%$ increased IgM expression on circulating B cells relative to wild-type B cells $(P<0.0001)$. Surface IgM levels on splenic B cells from TSK/+ mice were $10 \%$ lower than those of wild-type B cells $(P<0.05)$. By contrast, splenic B cells from $\mathrm{CD} 19^{-/-}$and $\mathrm{CD} 19^{-/-\mathrm{TSK}} /+$ mice had significantly elevated IgM expression levels compared with wild-type B cells (34\%, $P<0.001$, and $38 \%, P<0.0001$, respectively). CD23 expression levels, an indicator of B cell activation, on splenic B cells from TSK/+ mice were significantly $47 \%$ higher than those found in wild-type $\mathrm{B}$ cells $(P<0.005)$ but were normal on CD19-/-TSK/+ $\mathrm{B}$ cells. Expression levels of MHC class II antigens (I-A), CD44, CD54, and CD86 were similar for TSK/+ and wild-type B cells (data not shown). Thus, TSK/+ B cells exhibited the characteristic hyperresponsive phenotype due to abnormal basal signaling thresholds.

The effect of the TSK/+ mutation on B cell responsiveness was assessed by determining serum Ig levels in TSK/+ mice (Figure 4). TSK/+ mice had significantly elevated $\operatorname{IgM}(P<0.05), \operatorname{IgG} 1(P<0.05), \operatorname{IgG} 2 \mathrm{a}$ $(P<0.0005)$, and $\operatorname{IgG} 2 \mathrm{~b}(P<0.001)$ levels compared with those of wild-type littermates, while IgG3 and IgA levels were not significantly different from those of wild-type littermates. By contrast, $\mathrm{CD} 19^{-/-}$mice had significantly decreased IgG1 $(P<0.001)$, IgG3 $(P<0.0001)$, and IgA $(P<0.01)$ levels compared with wild-type littermates. Similarly, CD19-/-TSK/+ mice had significantly lower IgG1 and IgG3 concentrations compared with TSK/+ and wild-type littermates $(P<0.01)$, while levels of other isotypes were similar to those found in wild-type littermates. Thus, TSK/+ mice exhibited hyper- $\gamma$-globulinemia that was dependent on CD19 signal transduction.

CD19 loss abrogated autoantibody production in TSK/+ mice. ANAs in CD19-/-, TSK/+, CD19-/-TSK/+, and wildtype littermates were determined by indirect immunofluorescence staining using HEp-2 cells as the substrate. ANAs with a homogenous chromosomal staining pattern were detected in 33\% (10/30) of TSK/+ mice but were rarely detectable in $C D 19^{-/-}(6 \%, 1 / 16)$, CD19-/-TSK/+ $(6 \%, 1 / 16)$, and wild-type $(6 \%, 2 / 31)$ littermates. Since a homogenous staining pattern generally results from a variety of autoantibodies including Ab's against topo I, dsDNA, and histone, autoantibody specificities were assessed by ELISA (Figure 5). The dilution of sera giving half-maximal OD values in ELISAs was also determined to generate arbitrary units per milliliter that could be directly compared between mutant and wild-type littermates. Pooled sera from TSK/+ mice had mean IgM and IgG anti-topo I Ab levels that were 37- and 3.8-fold higher than those found in wild-type littermates $(P<0.01$ and $P<0.05$, respectively). TSK/+ mice had mean IgM Ab levels to dsDNA and histone that were 2.6-fold $(P<0.05)$ and 4.6-fold $(P<0.05)$ higher than those found in wild-type littermates, respectively, whereas IgG Ab levels to ssDNA, dsDNA, and histone were similar for TSK/+ and wildtype littermates. Mean IgM and IgG anti-Fbn-1 Ab

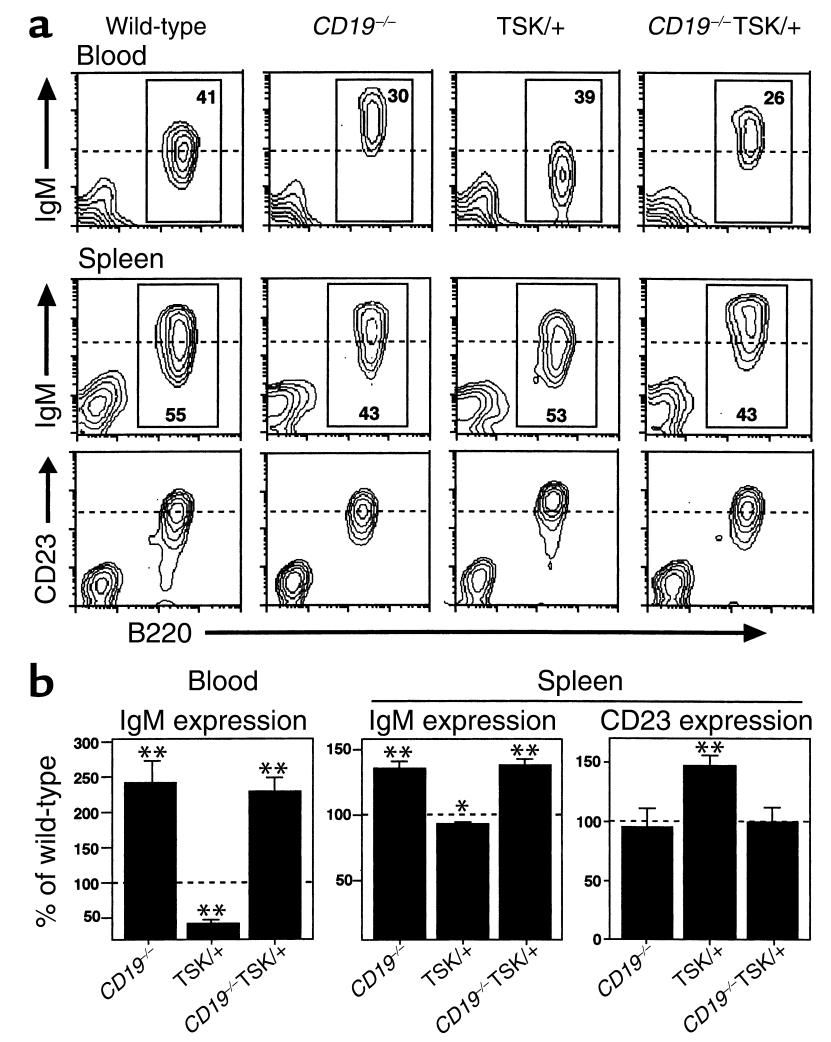

Figure 3

Cell surface IgM or CD23 expression and B cell development in mutant and wild-type littermates. (a) Representative two-color indirect immunofluorescence staining with flow cytometric analysis of $B$ cells. Values represent the percentage of the total gated lymphoid cell population that falls into the indicated gates. Horizontal dashed lines indicate mean IgM or CD23 expression of wild-type B cells to highlight differences in expression levels. These results represent those obtained with at least six mice of each genotype. (b) Relative cell surface IgM or CD23 densities were determined by comparing mean $( \pm$ SEM) linear fluorescence intensity channel numbers for immunofluorescence staining between $B$ cells from mice of each genotype ( $n=6$ for each). ${ }^{*} P<0.05,{ }^{*} P<0.01$ vs. wild-type littermates. 

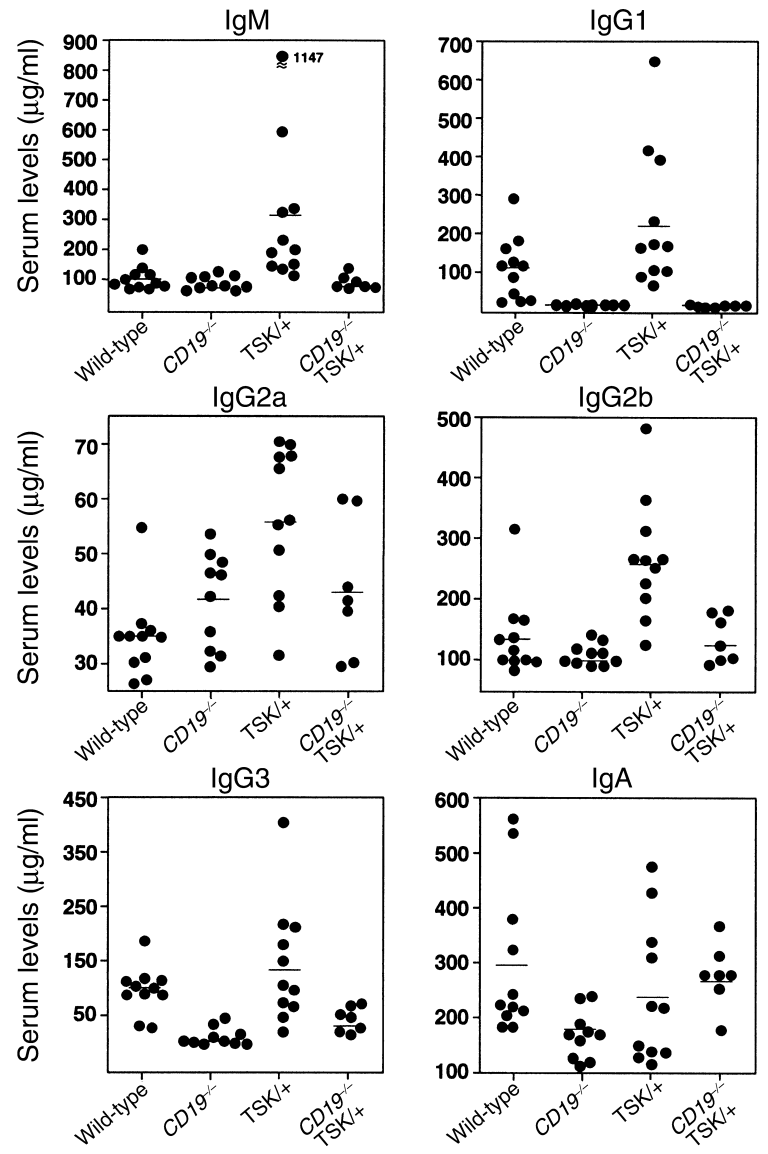

Figure 4

Serum Ig levels in mutant and wild-type littermates. Ig levels were determined by isotype-specific ELISAs. Horizontal bars represent mean Ig levels. Statistical analysis is provided in Results.

levels were significantly $52 \%(P<0.005)$ and 2.0 -fold $(P<0.05)$ higher in TSK $/+$ mice than those in wild-type littermates. TSK/+ mice also had rheumatoid factor $(\mathrm{RF})$ levels that were increased 3.3-fold relative to those of wild-type littermates $(P<0.01)$. By contrast, $C D 19^{-/-}$ and $C D 19^{-/-}$TSK/+ mice had decreased IgM and IgG Ab's reactive with topo I, ssDNA, dsDNA, histone, and Fbn-1 relative to those of wild-type littermates $(P<0.05)$, while $C D 19^{-/-}$and $C D 19^{-/-T S K} /+$ mice had RF levels similar to those of their wild-type littermates. Thus, loss of the CD19 signaling pathway dramatically inhibited autoantibody production in TSK $/+$ mice. $B$ cell development in TSK $/+$ and $C D 19^{-/-}$TSK $/+$mice. $\mathrm{B}$ cell development in the bone marrow was normal in TSK/+ mice (Table 1). Both the frequency and the number of blood, tissue, and peritoneal B cells were also normal in TSK/+ mice (Figure 3 and Table 1). Unexpectedly, however, TSK/+ mice had significantly decreased numbers of peritoneal $\mathrm{CD}^{+} \mathrm{B} 220^{\text {low }} \mathrm{B} 1$ cells compared with wild-type littermates (70\% reduction, $P<0.05)$, while $\mathrm{CD}^{-}{ }^{-} \mathrm{B} 220^{\mathrm{hi}}$ conventional $\mathrm{B} 2$ cell numbers were comparable with those of wild-type littermates. Thus, B cell development in TSK/+ mice was normal except for reduced peritoneal $\mathrm{B} 1$ cell development. By comparison, peripheral B cell numbers in $\mathrm{CD} 19^{-/-} \mathrm{TSK} /+$ mice were reduced, but similar to those normally found in $\mathrm{CD}_{19^{-/}}$ littermates, with a significant reduction $(\sim 90 \%, P<0.01)$ in peritoneal B1 cells. Thus, CD19 deficiency reduced the numbers of peripheral B cells in TSK/+ mice but did not severely limit B cell development.

CD19 loss attenuated the development of skin fibrosis in TSK $/+$ mice. Skin fibrosis in TSK/+, CD19-/-, CD19-/-TSK/+, and wild-type littermates was assessed by histopathology of the full-thickness skin sections from the back. The dermal thickness (the thickness from the top of the granular layer to the junction between the dermis and subcutaneous fat) was similar among TSK $/+(32 \pm 2 \mu \mathrm{m}), C^{-1} 9^{-/}(33 \pm 2 \mu \mathrm{m})$, CD19--TSK/+ $(33 \pm 2 \mu \mathrm{m})$, and wild-type $(34 \pm 3 \mu \mathrm{m})$ littermates (Figure 6a). The thickness of subcutaneous fat and panniculus carnosus was also similar among mutant and wild-type littermates (data not shown). Thus, increased skin fibrosis in TSK/+ mice was not due to hyperplasia of dermal connective tissue but resulted from hyperplasia of a subcutaneous loose connective tissue layer (i.e., the hypodermis or superficial fascia) beneath panniculus carnosus (Figure 6a). The hypodermal thickness in TSK/+ mice was increased ninefold compared with wild-type littermates $(P<0.0001$, Figure 6b). CD19-/-TSK/+ mice showed a moderate thickening of hypodermal tissue, which was significantly 36\% thinner than that found in TSK $/+$ mice $(P<0.001)$ but remained significantly thicker than that of wild-type littermates $(P<0.001)$. Hypodermal thickness was similar in wild-type and CD19-/- littermates.

Cutaneous fibrosis was also assessed by quantifying the hydroxyproline content of $6-\mathrm{mm}$ punch biopsies from skin samples of mutant and wild-type littermates (Figure 6c). Hydroxyproline content in TSK/+ mice was increased by $26 \%$ relative to that in wild-type littermates $(P<0.0005)$. CD19 deficiency reduced hydroxyproline content by $13 \%$ in TSK $/+$ mice $(P<0.01)$, but hydroxyproline content remained significantly higher than that in wild-type littermates $(P<0.01)$. Hydroxyproline content was comparable between wild-type and $C D 19^{-/-}$littermates. CD19 deficiency did not significantly affect the onset or severity of pulmonary emphysema and cardiac hypertrophy in TSK/+ mice (data not shown). Thus, B cells contributed to the induction of skin fibrosis in TSK/+ mice through a CD19-dependent pathway.

CD19 deficiency suppressed overproduction of IL-6 by $T S K /+B$ cells. It has been suggested that IL- 6 production contributes to skin fibrosis as well as the immunological abnormalities found in human SSc $(23,24)$. IL-6, a pleiotropic cytokine produced by B cells and various other cell types, induces the production of collagen and glycosaminoglycans by dermal fibroblasts, stimulates the proliferation and differentiation of $\mathrm{B}$ cells, and enhances $\mathrm{Ab}$ production (25). Therefore, IL-6 production by splenic B cells from mutant and wild-type littermates was assessed. Supernatant fluid from $B$ cell cultures contained only negli- 

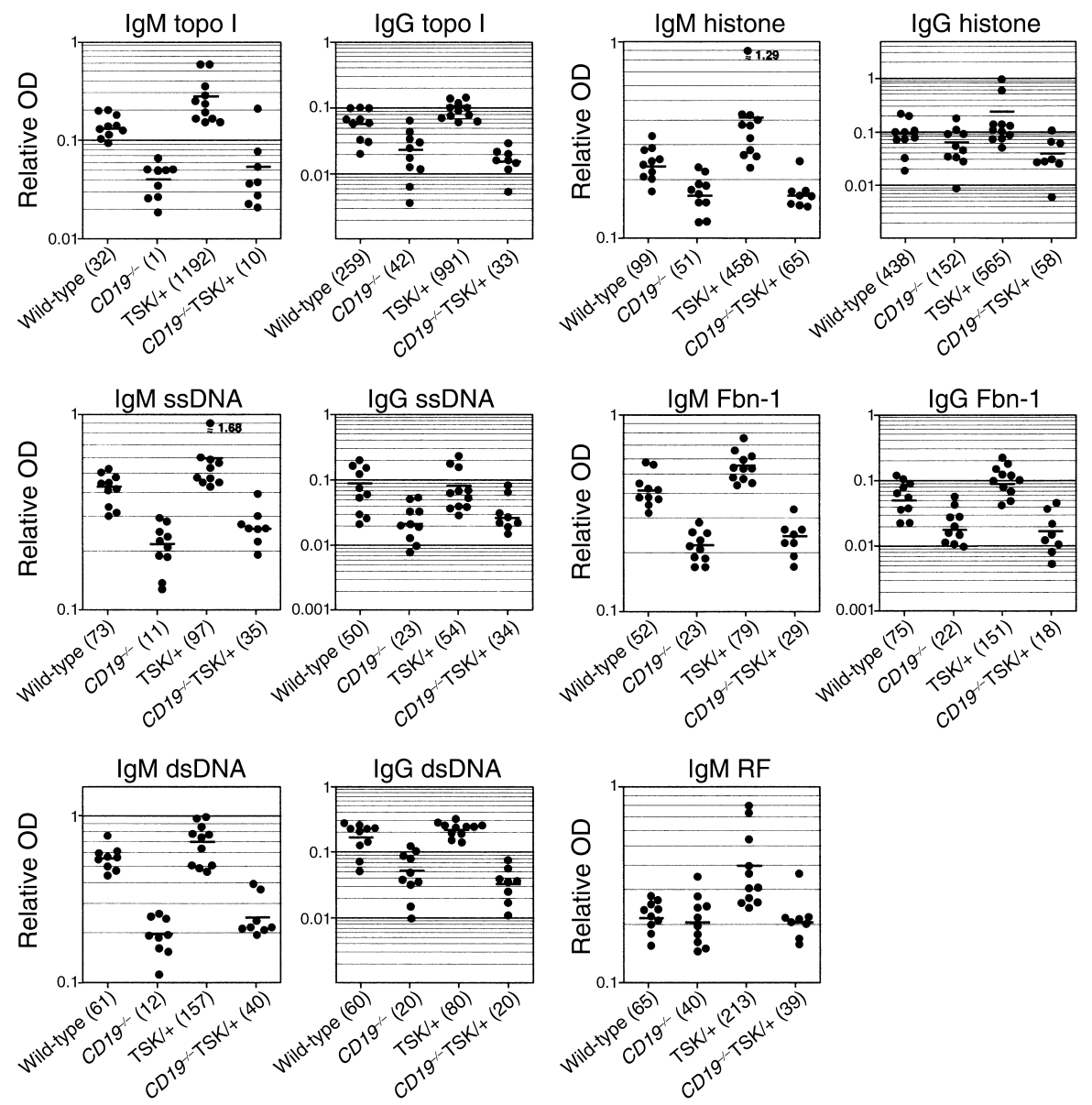

\section{Figure 5}

Anti-topo I, anti-ssDNA, anti-dsDNA, anti-histone, anti-Fbn-1, and RF Ab levels in sera from mutant and wildtype littermates. Relative autoantibody levels were determined by Ig subclass-specific ELISA. Horizontal bars represent mean autoantibody levels. Values in parentheses represent the dilutions of pooled sera giving halfmaximal OD values in autoantigenspecific ELISAs, which were determined by linear regression analysis to generate arbitrary units per milliliter that could be directly compared between each group of mice. Statistical analysis is provided in Results. gible quantities of IL-6 (Figure 7a). By contrast, TSK/+ $\mathrm{B}$ cells stimulated with anti-IgM plus anti-CD40 mAb's produced amounts of IL- 6 that were $78 \%$ higher than those found in cultures of stimulated wild-type B cells $(P<0.05)$. CD19 deficiency significantly inhibited IL- 6 production by TSK $/+$ B cells $(55 \%, P<0.05)$ but did not inhibit IL- 6 production by wild-type B cells. Thus, IL- 6 production was augmented in TSK/+ $B$ cells through a CD19-dependent signaling pathway.

CD19 deficiency inbibited IL-4-induced IgG1 overproduction by TSK $/+B$ cells. It has been demonstrated that IL-4 plays a critical role in the cutaneous hyperplasia of TSK/+ mice, since deficiency of IL- 4 or IL- 4 receptor $\alpha$ (IL-4R $\alpha$ ) inhibits skin fibrosis and autoantibody production $(17,26)$. IL-4 activates B cells that secrete IgG1 in vitro in the presence of LPS. Therefore, responsiveness of TSK/+ B cells to IL-4 was assessed by IgG1 production of splenic B cells. Background levels of IgG1 secretion were similar for mutant and wild-type littermates (Figure 7b). Stimulation of TSK/+ B cells with IL-4 plus LPS resulted in IgG1 secretion that was 38\% higher than that of wild-type B cells $(P<0.05)$. The CD19 loss in TSK/+ B cells inhibited IgG1 production (40\%, $P<0.05)$. However, IgG1 production by CD19-/$\mathrm{B}$ cells was normal as previously reported (27). Thus, TSK/+ B cells exhibited enhanced activation by IL-4 that was eliminated by CD19 deficiency.

\section{Discussion}

The current study demonstrates that B cells are critical for the development of skin fibrosis in the TSK model of SSc (Figure 6). Specifically, B cells in TSK/+ mice displayed a hyperresponsive phenotype characterized by downregulated surface IgM expression and upregulated CD23 expression (Figure 3), constitutively enhanced CD19 and Vav tyrosine phosphorylation (Figure 1, a and b), augmented Lyn kinase activity (Figure 1c), increased CD19-induced $\left[\mathrm{Ca}^{2+}\right]_{i}$ responses (Figure $2 \mathrm{a}$ ), and hyper$\gamma$-globulinemia (Figure 4). TSK/+ B cells also exhibited augmented proliferative response to BCR ligation (Figure $2 \mathrm{~b}$ ). However, B cell surface receptor-mediated signaling was not globally abnormal, since proliferation in response to LPS or anti-CD38 $\mathrm{mAb}$ was normal in TSK/+ B cells. Most significant, however, was that the hypodermal thickness and collagen deposition of skin were significantly decreased in TSK/+ mice due to CD19 deficiency (Figure 6). In addition, autoantibody levels (Figure 5), IL-6 production by activated B cells (Figure 7a), and IgG1 secretion by B cells activated with IL-4 (Figure 7b) were reduced in TSK/+ mice by CD19 deficiency. Thus, a CD19-dependent signaling pathway in B cells contributes to the development of systemic autoimmunity and skin sclerosis in TSK/+ mice.

Consistent with a role for $\mathrm{B}$ cells in $\mathrm{TSK} /+$ mice, adoptive transfer of both $\mathrm{T}$ and $\mathrm{B}$ cells from $\mathrm{TSK} /+$ 


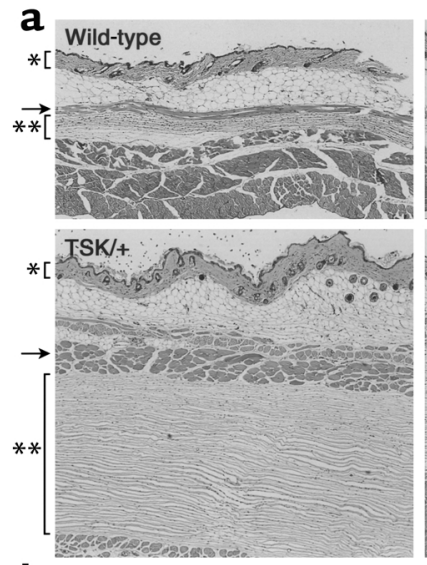

b
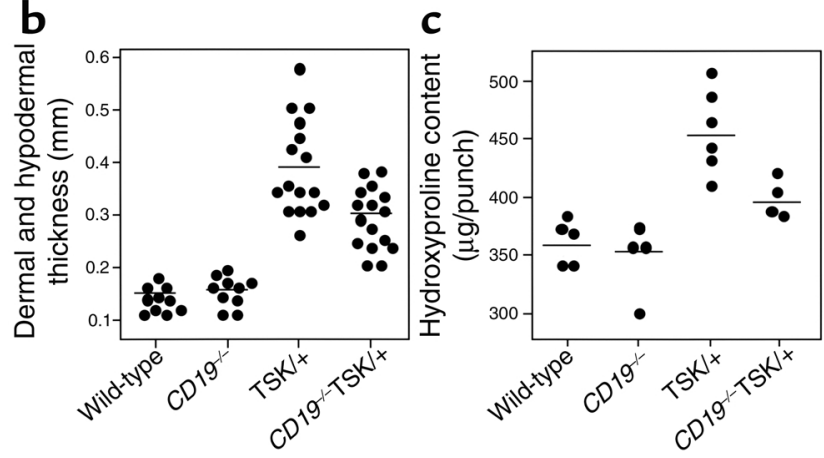

mice induces cutaneous collagen deposition and autoantibody production in wild-type mice, while the infusion of purified $\mathrm{T}$ cells does not lead to the development of TSK syndrome (28). In addition, the development of skin fibrosis correlates closely with serum anti-topo I Ab levels in TSK/+ mice (29). Although Kasturi et al. have reported that mature $\mathrm{B}$ cell loss does not abrogate dermal thickening in TSK/+ mice (6), this difference in conclusions may result from the measurement of different parameters. The current study (Figure 6$)$ and other previous studies $(4,30-32)$ have shown

\section{Figure 6}

Skin fibrosis in dorsal skin from mutant and wild-type littermates. (a) Representative histologic sections stained with hematoxylin and eosin are shown $(\times 40)$. An asterisk indicates the dermis, and double asterisks show the subcutaneous loose connective tissue layer (i.e., the hypodermis or superficial fascia) beneath the panniculus carnosus (arrow). These results represent those obtained with at least ten mice of each genotype. Skin fibrosis was assessed by quantitatively measuring hypodermal thickness (b) and skin hydroxyproline content (c). The hypodermal thickness was measured under a light microscope as the thickness of the hypodermis or superficial fascia beneath the panniculus carnosus. The quantity of hydroxyproline is expressed as $\mu \mathrm{g}$ per $6-\mathrm{mm}$ punch biopsy. Horizontal bars represent mean hypodermal thickness (b) and mean hydroxyproline content (c). Statistical analysis is provided in Results.

that dermal thickness is similar in TSK/+ and wild-type littermates, but the hypodermal thickness, including a subcutaneous connective tissue layer, is significantly increased in TSK/+ mice relative to wild-type littermates (4, 30-32). In fact, Green et al. originally characterized the cutaneous pathology in TSK $/+$ mice as a massive increase in the subcutaneous tissue with no obvious changes present in the dermis and epidermis (4). Another study has reported that TSK/+ BALB/c mice deficient in both $\mathrm{B}$ and $\mathrm{T}$ cell function develop cutaneous fibrosis that is indistinguishable from that found in TSK/+ mice (31). This result conflicts not only with the current study but also with previous studies where immunocompetent cells contributed to the development of skin fibrosis in TSK/+ mice (28, 32 ). However, the BALB/c genetic background may affect cutaneous fibrosis in TSK/+ mice, since the hypodermal thickness of TSK/+ BALB/c mice is only twofold increased compared with the nine- to tenfold increase observed on a C57BL/6 background (Figure 6) (30). Thus, the contribution of B cells to the pathogenesis of TSK syndrome may not be consistent across all genetic backgrounds.

Table 1

B lymphocyte development in CD19-/-, TSK/+, CD19-/-TSK/+, and wild-type littermates

Frequency $(\%)$ and number $\left(\# 10^{-6}\right)$ of B cells

\begin{tabular}{|c|c|}
\hline Tissue & \\
\hline Bone marrow & $\begin{array}{l}\% \operatorname{lgM}^{-} B 220^{\text {low }} \\
\% \operatorname{lgM}^{+} B 220^{\text {low }} \\
\% \operatorname{lgM}^{+} B 220^{\text {hi }}\end{array}$ \\
\hline Blood & $\begin{array}{l}\% \operatorname{lgM}^{+} \text {B2220+ } \\
\# \operatorname{lgM}^{+} \mathrm{B}^{+} 20^{+}\end{array}$ \\
\hline Spleen & $\begin{array}{l}\% \operatorname{lgM}^{+} \text {B220 } 20^{+} \\
\# \operatorname{lgM}^{+} \text {B2220 }\end{array}$ \\
\hline Peritoneal cavity & 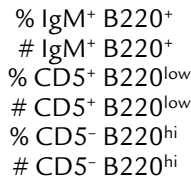 \\
\hline
\end{tabular}

\section{Wild-type}

$40.3 \pm 3.0$

$24.1 \pm 4.4$

$15.2 \pm 1.2$

$40.7 \pm 2.3$

$2.2 \pm 0.4$

$56.8 \pm 0.9$

$50.2 \pm 3.4$

$56.2 \pm 2.6$

$1.4 \pm 0.2$

$32.9 \pm 2.1$

$0.9 \pm 0.3$

$23.6 \pm 0.9$

$0.7 \pm 0.2$

\section{CD19-/-}

$34.9 \pm 4.5$

$25.1 \pm 3.1$

$10.2 \pm 2.1$

$30.8 \pm 1.1^{\mathrm{A}}$

$2.1 \pm 0.4$

$40.0 \pm 3.3^{\mathrm{A}}$

$31.4 \pm 3.1^{\mathrm{B}}$

$27.6 \pm 5.5^{\mathrm{A}}$

$0.5 \pm 0.1^{\mathrm{B}}$

$10.1 \pm 1.0^{\mathrm{A}}$

$0.1 \pm 0.1^{\mathrm{A}}$

$24.8 \pm 4.0$

$\begin{array}{r}0.3 \pm 0.1^{\mathrm{B}} \\ \hline\end{array}$

\section{TSK/+}

$40.3 \pm 2.4$

$24.2 \pm 4.7$

$13.3 \pm 1.8$

$44.9 \pm 2.0$

$3.0 \pm 0.5$

$57.1 \pm 2.2$

$54.4 \pm 2.6$

$67.1 \pm 3.4^{\mathrm{A}}$

$1.1 \pm 0.3$

$17.0 \pm 2.7^{\mathrm{A}}$

$0.3 \pm 0.1^{\mathrm{A}}$

$49.5 \pm 4.0^{\mathrm{B}}$

$0.8 \pm 0.2$
CD19-/-TSK/+

$35.7 \pm 4.5$

$21.7 \pm 2.5$

$11.7 \pm 1.6$

$26.7 \pm 3.7 \mathrm{~B}$

$1.8 \pm 0.4$

$37.4 \pm 2.3^{\mathrm{A}}$

$32.2 \pm 3.1^{\mathrm{B}}$

$26.7 \pm 7.7^{\mathrm{A}}$

$0.3 \pm 0.1^{\mathrm{A}}$

$6.8 \pm 0.2^{\mathrm{A}}$

$0.1 \pm 0.1^{\mathrm{A}}$

$34.4 \pm 16.3$

$0.5 \pm 0.2$

Values represent the number or percentage $( \pm \mathrm{SD}, n=5)$ of lymphocytes (based on side and forward light scatter properties) expressing the indicated cell surface markers. The background percentage of cells that were positive $(<1 \%)$ was subtracted from the values shown. ${ }^{A, B}$ The number or percentage of cells was significantly different from that of wild-type littermates: ${ }^{A} P<0.01,{ }^{B} P<0.05$. 
The present study suggests that B cells play an important role not only in autoantibody production, but also in the development of cutaneous fibrosis itself. Indeed, critical roles of B cells in disease expression have also been suggested in animal models of other collagen diseases. Elimination of B cells in lupus-prone mice results in a complete abrogation of glomerulonephritis, vasculitis, and skin disease (33). Furthermore, lupus-prone mice with B cells that cannot secrete Ab's still develop nephritis and vasculitis (33). This finding indicates that, independent of autoantibody, B cells are essential for disease expression, either by serving as antigen-presenting cells or by contributing directly to local inflammation through cytokine secretion. Pathogenic autoantibodies from $\mathrm{B}$ cells are also important, since $\mathrm{K} / \mathrm{BxN}$ mice, a model for human rheumatoid arthritis, have hyperactive $B$ cells that cause hyper- $\gamma$-globulinemia and produce arthritogenic autoantibody (34). Collectively, B cells are crucial for development of autoimmunity and disease expression in systemic autoimmune models.

Although autoantibody production is a major characteristic of TSK/+ mice, intrinsic B cell abnormalities have not, to our knowledge, been previously identified. We believe that the present study is the first to reveal augmented B cell function in TSK/+ mice and the first to demonstrate that autoimmunity in TSK $/+$ mice is dependent on CD19 signaling. The increase in constitutive CD19 and Vav tyrosine phosphorylation and Lyn kinase activity in TSK/+ B cells (Figure 1) undoubtedly increases the formation and activity of CD19/Lyn/Vav complexes, which lowers base-line signaling thresholds required for $B$ cell activation $(10,19,20)$. This is consistent with enhanced BCR-mediated proliferation by TSK/+ B cells (Figure 2b), since CD19 augments BCR signaling $(10,16,22)$. Downregulation of surface IgM expression on B cells in TSK/+ mice (Figure 3 ) is similar to what has been previously shown in other autoimmune strains of mice such as transgenic mice overexpressing CD19, CD22-/- mice, and SHP-1 defective $m e^{v} / m e^{v}$ mice $(18,21,22)$. Taken together, these results indicate that the $\mathrm{B}$ cell abnormalities observed in TSK/+ mice are due in part to augmented signaling through a CD19-dependent pathway.

Although skin fibrosis and autoimmunity in TSK/+ mice were significantly inhibited in the absence of CD19 expression (Figures 5 and 6), the development of lung emphysema and myocardial hypertrophy were not affected (data not shown). These findings demonstrate that TSK disease is likely to be multigenic, rather than due to a single mutation in the Fbn-1 gene. In further support of this, CD4 deficiency in TSK/+ mice results in decreased cutaneous fibrosis but does not affect lung emphysema or anti-topo I Ab levels (32). Some studies also suggest that the abnormal microfibrils resulting from the duplicated Fbn-1 gene are associated only with the development of pulmonary emphysema, and not with tissue hyperplasia and autoimmunity (7). Other studies suggest that the mutated Fbn-1 gene contributes to skin fibrosis and autoimmunity, but not to pulmonary emphysema and cardiac hypertrophy (9). Thus, the relationship of the duplicated Fbn-1 gene to autoimmunity and hyperplasia of skin and internal organs remains unclear, although additional genetic defects that affect CD19 function in B cells are likely to exist in linkage with the duplicated Fbn-1 gene in TSK mice.

In human SSc, B cells overexpress CD19 (15). In addition, various growth factors and cytokines that are partly produced by immune cells have been suggested to play central roles in the initiation and development of fibrosis. Since TSK/+ B cells were chronically activated in vivo (Figures 1 and 3 ), it can be hypothesized that cytokines produced by activated B cells may contribute to the induction of skin fibrosis in TSK/+ mice. Consistent with this, TSK/+ B cells produced more IL-6 than did wild-type B cells in response to BCR and CD40 ligation (Figure 7a). Enhanced IL-6 production by $\mathrm{TSK} /+\mathrm{B}$ cells was also eliminated by a loss in CD19 expression. IL-6 induces concentration-dependent increases in the production of collagen and glycosaminoglycans by dermal fibroblast (25). Serum IL-6 levels are higher in patients with SSc than in normal controls, and IL- 6 production by cultured PBMCs from patients with SSc is augmented relative to normal controls (23). Furthermore, function-blocking anti-IL-6 Ab's significantly inhibit the production of procollagen type I in cultured SSc fibroblasts (24). Moreover, Vavinduced Rac1 activation leads to the production and subsequent autocrine action of IL-6 (35). Therefore, enhanced Vav tyrosine phosphorylation downstream of CD19 in TSK/+ B cells (Figure 1b) may result in IL-6 overproduction that contributes in part to skin fibrosis in TSK/+ mice. Recent studies have demonstrated
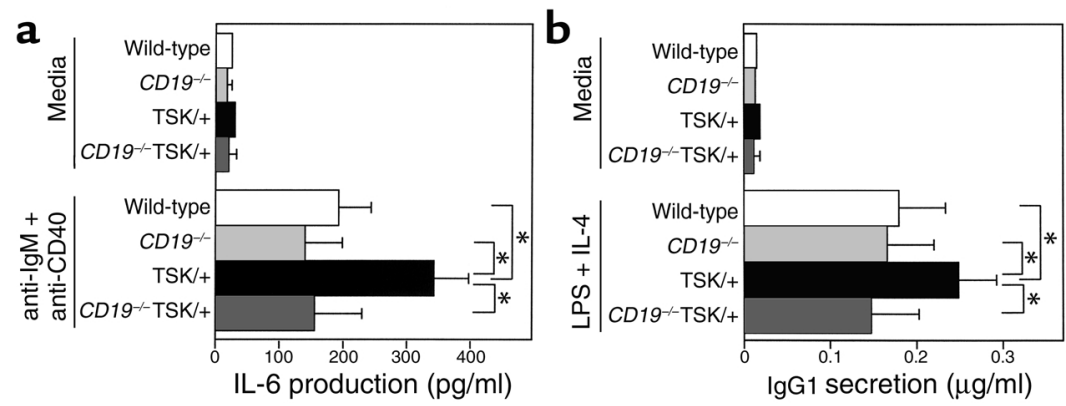

\section{Figure 7}

Hyperresponsiveness of $B$ cells from $T S K /+$ mice. (a) IL-6 production by $B$ cells from mutant and wild-type littermates. Spleen B cells were stimulated with either media alone or anti-IgM plus antiCD40 mAb's for 48 hours. (b) IgG1 secretion by B cells stimulated with IL-4. Spleen B cells were cultured with either media alone or IL-4 plus LPS for 5 days. Cell supernatants were analyzed by ELISA to determine the amount of secreted IL-6 or IgG1. Each histogram shows the mean $( \pm S D)$ results obtained for six mice of each genotype. ${ }^{*} P<0.05$. 
the importance of IL-4 for the development of cutaneous fibrosis, since deficiency of IL-4R $\alpha$ or IL- 4 inhibited skin fibrosis and autoantibody production $(17,26)$. In the current study, TSK/+ B cells exhibited hyperresponsiveness to IL-4 that was eliminated by CD19 deficiency (Figure $7 \mathrm{~b}$ ). Therefore, it is also possible that the hyperresponsiveness of TSK/+ B cells to IL-4 may contribute to skin fibrosis and autoimmunity.

The presence of autoantibodies is a central feature of human SSc, since specific autoantibodies are detected in more than $90 \%$ of patients. In addition, anti-topo I Ab levels closely correlate with disease activity and severity in human SSc. However, the pathogenic relationship between systemic autoimmunity and the clinical manifestations of SSc including skin fibrosis remains unknown, since autoantigens are generally intracellular components critical for cell mitosis and autoantibodies are not thought to contribute to tissue damage in SSc. The current study indicates that autoantibody production is linked to skin sclerosis through chronic B cell activation that results from constitutively enhanced CD19 signaling. Alternatively, the link between skin fibrosis and autoimmunity may be the presence in $\mathrm{TSK} /+$ mice of anti-Fbn-1 autoantibodies that were eliminated by CD19 deficiency (Figure 5), since normal fibroblasts treated with anti-Fbn-1 autoantibodies display an activated phenotype overexpressing Fbn-1 as well as some other ECM components (36). Taken together, the present study suggests that B cells or cell surface regulatory molecules on B cells, including CD19, may be possible targets in the therapy of SSc.

\section{Acknowledgments}

This work was supported by a Grant-in-Aid from the Ministry of Education, Science, and Culture of Japan (to S. Sato and M. Fujimoto) and NIH grants CA-81776 and CA-54464 (to T.F. Tedder). We thank M. Matsubara and Y. Yamada for technical assistance.

1. Bona, C., and Rothfield, N. 1994. Autoantibodies in scleroderma and tightskin mice. Curr. Opin. Immunol. 6:931-937.

2. Murai, C., Saito, S., Kasturi, K.N., and Bona, C.A. 1998. Spontaneous occurrence of anti-fibrillin-1 autoantibodies in tight-skin mice. Autoimmunity. 28:151-155.

3. Tan, F.K., et al. 1999. Autoantibodies to the extracellular matrix microfibrillar protein, fibrillin-1, in patients with scleroderma and other connective tissue diseases. J. Immunol. 163:1066-1072.

4. Green, M.C., Sweet, H.O., and Bunker, L.E. 1976. Tight-skin, a new mutation of the mouse causing excessive growth of connective tissue and skeleton. Am. J. Pathol. 82:493-512.

5. Siracusa, L.D., et al. 1996. A tandem duplication within the fibrillin 1 gene is associated with the mouse tight skin mutation. Genome Res. 6:300-131.

6. Kasturi, K.N., et al. 1997. B-cell deficiency does not abrogate development of cutaneous hyperplasia in mice inheriting the defective fibrillin-1 gene. J. Autoimmun. 10:505-517.

7. Gayraud, B., Keene, D.R., Sakai, L.Y., and Ramirez, F. 2000. New insights into the assembly of extracellular microfibrils from the analysis of the fibrillin 1 mutation in the tight skin mouse. J. Cell Biol. 150:667-680.

8. Kielty, C.M., et al. 1998. The Tight skin mouse: demonstration of mutant fibrillin-1 production and assembly into abnormal microfibrils. J. Cell Biol. 140:1159-1166.

9. Saito, S., et al. 2000. Induction of skin fibrosis in mice expressing a mutated fibrillin-1 gene. Mol. Med. 6:825-836.

10. Tedder, T.F., Inaoki, M., and Sato, S. 1997. The CD19/21 complex regulates signal transduction thresholds governing humoral immunity and autoimmunity. Immunity. 6:107-118.
11. Hibbs, M.L., et al. 1995. Multiple defects in the immune system of Lyndeficient mice, culminating in autoimmune disease. Cell. 83:301-311.

12. Painter, C.J., et al. 1988. Specificities and V genes encoding monoclonal autoantibodies from viable motheaten mice. J. Exp. Med. 167:1137-1153.

13. Sato, S., Ono, N., Steeber, D.A., Pisetsky, D.S., and Tedder, T.F. 1996. CD19 regulates $\mathrm{B}$ lymphocyte signaling thresholds critical for the development of B-1 lineage cells and autoimmunity. J. Immunol. 156:4371-4378.

14. Inaoki, M., Sato, S., Weintraub, B.C., Goodnow, C.C., and Tedder, T.F. 1997. CD19-regulated signaling thresholds control peripheral tolerance and autoantibody production in B lymphocytes. J. Exp. Med. 186:1923-1931.

15. Sato, S., Hasegawa, M., Fujimoto, M., Tedder, T.F., and Takehara, K. 2000 Quantitative genetic variation in CD19 expression correlates with autoimmunity. J. Immunol. 165:6635-6643.

16. Engel, P., et al. 1995. Abnormal B lymphocyte development, activation and differentiation in mice that lack or overexpress the CD19 signal transduction molecule. Immunity. 3:39-50.

17. McGaha, T., et al. 2001. Lack of skin fibrosis in tight skin (TSK) mice with targeted mutation in the interleukin-4R $\alpha$ and transforming growth factor- $\beta$ genes. J. Invest. Dermatol. 116:136-143.

18. Sato, S., et al. 1996. CD22 is both a positive and negative regulator of B lymphocyte antigen receptor signal transduction: altered signaling in CD22-deficient mice. Immunity. 5:551-562.

19. Fujimoto, M., Poe, J.C., Jansen, P.J., Sato, S., and Tedder, T.F. 1999. CD19 amplifies B lymphocyte signal transduction by regulating Src-family protein tyrosine kinase activation. J. Immunol. 162:7088-7094.

20. Fujimoto, M., et al. 2000. CD19 regulates Src family protein tyrosine kinase activation in B lymphocytes through processive amplification. Immunity. 13:47-57.

21. Cyster, J.G., and Goodnow, C.C. 1995. Protein tyrosine phosphatase 1C negatively regulates antigen receptor signaling in B lymphocytes and determines thresholds for negative selection. Immunity. 2:13-24.

22. Sato, S., Steeber, D.A., Jansen, P.J., and Tedder, T.F. 1997. CD19 expression levels regulate B lymphocyte development: human CD19 restores normal function in mice lacking endogenous CD19. J. Immunol. 158:4662-4669.

23. Hasegawa, M., Sato, S., Ihn, H., and Takehara, K. 1999. Enhanced production of interleukin-6 (IL-6), oncostatin M and soluble IL-6 receptor by cultured peripheral blood mononuclear cells from patients with systemic sclerosis. Rheumatology. 38:612-617.

24. Kawaguchi, Y., Hara, M., and Wright, T.M. 1999. Endogenous IL-1 $\alpha$ from systemic sclerosis fibroblasts induces IL-6 and PDGF-A. J. Clin. Invest. 103:1253-1260.

25. Duncan, M.R., and Berman, B. 1991. Stimulation of collagen and glycosaminoglycan production in cultured human adult dermal fibroblasts by recombinant human interleukin 6. J. Invest. Dermatol. 97:686-692.

26. Kodera, T., McGaha, T.L., Phelps, R., Paul, W.E., and Bona, C.A. 2002. Disrupting the IL-4 gene rescues mice homozygous for the tight-skin mutation from embryonic death and diminishes TGF-beta production by fibroblasts. Proc. Natl. Acad. Sci. USA. 99:3800-3805.

27. Sato, S., Steeber, D.A., and Tedder, T.F. 1995. The CD19 signal transduction molecule is a response regulator of B-lymphocyte differentiation. Proc. Natl. Acad. Sci. USA. 92:11558-11562.

28. Phelps, R.G., Daian, C., Shibata, S., Fleischmajer, R., and Bona, C.A. 1993. Induction of skin fibrosis and autoantibodies by infusion of immunocompetent cells from tight skin mice into C57BL/6 Pa/Pa mice. J. Autoimmun. 6:701-718.

29. Hatakeyama, A., Kasturi, K.N., Wolf, I., Phelps, R.G., and Bona, C.A. 1996. Correlation between the concentration of serum anti-topoisomerase I autoantibodies and histological and biochemical alterations in the skin of tight skin mice. Cell. Immunol. 167:135-140.

30. Everett, E.T., Pablos, J.L. Harley, R.A., LeRoy, E.C., and Norris, J.S. 1995 The role of mast cells in the development of skin fibrosis in tight-skin mutant mice. Comp. Biochem. Physiol. 110A:159-165.

31. Dodig, T.D., Mack, K.T., Cassarino, D.F., and Clark, S.H. 2001. Development of the tight-skin phenotype in immune-deficient mice. Arthritis Rheum. 44:723-727.

32. Wallace, V.A., et al. 1994. A role for $\mathrm{CD}^{+} \mathrm{T}$ cells in the pathogenesis of skin fibrosis in tight skin mice. Eur. J. Immunol. 24:1463-1466.

33. Shlomchik, M.J., Craft, J.E., and Mamula, M.J. 2001. From T to B and back again: positive feedback in systemic autoimmune disease. Nat. Rev. Immunol. 1:147-153.

34. Korganow, A.S., et al. 1999. From systemic T cell self-reactivity to organspecific autoimmune disease via immunoglobulins. Immunity. 10:451-461.

35. Faruqi, T.R., Gomez, D., Bustelo, X.R., Bar-Sagi, D., and Reich, N.C. 2001 Rac1 mediates STAT3 activation by autocrine IL-6. Proc. Natl. Acad. Sci. USA. 98:9014-9019.

36. Zhou, X., et al. 2001. Anti-fibrillin-1 autoantibodies induce increased gene expression of FBN1 in normal dermal fibroblasts. Arthritis Rheum. 44:S194. (Suppl.) 